III.

\title{
Schwierigkeiten und Irrtümer bei der Diagnose eitriger Meningitis ex otitide.
}

(Nach einer Mitteilung auf dem 8 . internationalen Otologenkongre $B$ in Budapest)

von H. Schwartze.

Angesichts des sehr sorgfältigen und umfangreichen Referates, welches Dr. Lermoyez (Paris) uns geliefert hat, der leider nicht hier ersehienen ist, könnte man den Eindruck gewinnen, als könne es keine Schwierigkeiten bei der Diagnose mehr geben und seien Trrtümer bei derselben kaum noch möglieh. Für typisch verlaufende Fälle mag dies zutreffen, besonders seit dem Bekanntsein der Lumbalpunktion (H. Q $\mathrm{n}$ inke). Die Wiehtigkeit der letzteren für uns Otologen habe ich zuerst anf der 73. Versammlung deutseher Naturforscher und Ärzte in Hamburg 1901 bervorgehoben, und ihre diagnostis che Bedeutung ist seitdem immer allgemeiner anerkannt worden. Vereinzelte abfällige Urteile darüber (z. B. auch von Hein $\mathrm{e}^{1}$ ), welche die Punktion für bedenklich und bei der Diagnose fïr überflissig erklärten, basierten auf unzureichender Erfahrung, konnten aber nicht irre führen und sind jetzt verstummt. Ich glaube, es gibt heute wobl keinen Otologen mebr, der bei zweifelhafter Diagnose einer intrakraniellen Komplikation der Otitis die Lumbalpunktion als diagnostisches Hilfsmittel entbehren möchte. H. Quinke hat sich sicherlich durch die Einfthrung der Lumbalpunktion in die Diagnostik ein grobes und bleibendes Verdienst, auch um die Otologie erworben.

Aber auch dureh das Resultat der Lumbalpunktion können wir getäuscht werden, ganz abgesehen von den Fällen, wo sie tuberhaupt versagt (trocken bleibt, wie man sagt). Es bleibt also auch jetzt noch eine Zahl von Fällen übrig, wo die Diagnose

1) S. dieses Archiv, Bd. 50. S. 276. 
unsicher ist, dies ganz besonders in jenen Fällen, wo anderweitige Komplikationen der Otitis, intrakranielle oder extrakranielle, gleichzeitig bestehen. Dies sind aber gerade die für die Praxis wichtigsten Fälle, weil es sich dabei um die Frage handeln kann, ob eine Operation noch mit Aussicht auf günstigen Erfolg unternommen werden kann oder nicht.

Anschließend an einen eklatanten Fall falscher Diagnose auf Meningitis purulenta ex otitide, der bei mir in der Klinik im Wintersemester $1907 / 08$ vorkam, will ich einige Worte iber die Ursachen der möglichen Irrtümer sagen.

Zunächst der Fall.

Frida Schanz, 5 Jahre alt, aus Tollwitz bei Dürrenberg (aufgenommen am 20. Januar 1908). bekam nach diphteritischer Angina Anfang Januar d. J. akute Eiterung des linken Mittelohres. Schon nach wenigen Tagen sistierte die Eiterung und es war anscheinend Gesundung erfolgt. Seit dem 9. Januar erkrankt das Kind von neuem mit Appetitlosigkeit, Mattigkeit, öfterem Erbrechen, Benommenheit des Sensoriums. Nachdem diese Symptome 11 Tage bestanden batten mit Fieber, wurde das Kind am 20. Januar in unsere stationäre Klinik aufgenommen. Das Kind war sehr blaß, somnolent, so tief benommen, daß es weder auf Händeklatschen, noch auf Anschreien reagierte, zeigte dabei aber große motorisehe Unruhe. Der Kopf lag zuritekgezogen; bei Berührung im Genick äußerte das Kind lebhaften Schmerz; Temp. am Aufnahmetage bis $39,2^{\circ}$, am folgenden Tage bis 40,2 .

Ohrenbefund: R. normal. L. Gehörgang weit. L. Trommelfell ziemlich stark injiziert, seine Grenze gegen den Gehörgang nach hinten oben verstrichen. Starke Vorwölbung im hintern obern Quadranten des Trommelfelles durch Exsudatanbäufung dahinter. Hammergriff noch erkennbar.

A u genbefund: Pupillen mittelweit, gleich, von normaler Reaktion gegen Lichteinfall. Keine nachweisbaren Augenmuskelstörungen. Hohe Stau ungspapille links; ob reehts auch, ist nicht zu entsoheiden wegen parazentraler Hornhauttrübung und wegen der motorisehen Unruhe des Kindes. Sicher bestand aber auch rechts Neuritis optica. Es wurde sofort die Paracentese des linken Trommelfells gemacht und die Lumbalpunktion. Erstere entleerte aus der Paukenhöhle nur rein seröses Exsudat, letztere ea. $30 \mathrm{ccm}$ absolut klaren Liquor cerebrospinalis, der unter sehr starkem Drucke stand. Die Punktionsstelle am 
Wirbelkanal wurde wie gewöhnlich mit Englischpflaster und Kollodium verklebt. Am Tage nach der Paracentese und der Lumbalpunktion war eine eklatante Besserung im Befinden des Kindes eingetreten; das Sensorium war kaum noch benommen und besserte sich dann schnell fortschreitend. Eine sehr auffällige Änderung im ophthalmoskopischen Bilde trat gleichfalls schon am nächsten Tage nach der Lumbalpunktion hervor. Die Staungspapille warfastvölligzurüekgegangen. Statt dessen bot die Papille jetzt das Bild einer leichten Neuritis, die sich innerhalb 8 Tagen völlig zurückbildete.

Eine Erkläruug für diese ungewöhnliche Erseheinung gab vielleicht die Tatsache, dab 3 Tage nach der Lumbalpunktion andauernd noch Liquor cerebrospinalis a s der Stichstelle aussickerte, und zwar in solcher Menge, daß ein dicker Watteverband und die Bettunterlagen durchnäßt wurden und mehrfach gewechselt werden muften. Ein derartiges Nachsickern hatte ieh bei den zahlreichen Lumbalpunktionen, die auf meiner Klinik seit dem Bekanntwerden derselben gemacht wurden, niemals beobachtet. Einige Tage später hörte dann der Abfluß des Liquor allmählich vollständig auf.

$\mathrm{Ob}$ es nieht möglich wäre, diesen Weg der Danerdrainage aus der Stichöffnung, welchen hier die Natur eingeschlagen hat, um die schnelle Rüekbildung der cerebralen Erkrankung und der Stauungspapille herbeizuftuhren, artefiziell zu schaffen? Vielleicht ließe sich dadurch anch der therapeutische Wert der Lumbalpunktion erhöhen.

Aus dem Einschnitt in das Trommelfell hatte sich nur einen Tag lang seröser Eiter entleert. Dann war das Ohr dauernd trocken geblieben, und der Schnitt zeigte sich verklebt. Vom 26. Januar an dauerndes Wohlbefinden. Am 6. Februar konnte das Kind als völlig geheilt entlassen werden.

Die auf Meningitis purulenta gestellte Diagnose war also sicher falsch gewesen. Wer an eine Meningitis serosa glaubt, wird geneigt sein, eine solche hier anzunehmen. Die Möglichkeit der Existenz einer solchen Hirnhautentzindung mit rein serösem Exsudat muB ja theoretisch nach Analogie anderer seröser Häute zugeben werden. Der anatomische Nachweis einer solehen ist aber bisher nicht gefülirt, weil sie eben nicht zum Tode führte. Was gewöhnlich dafür gilt, ist Staungs-Hydrocephalus und Hirnödem. Leider wurde hier der dureh Punktion entleerte Liquor cerebrospinalis auf seinen Eiweilgehalt 
nicht untersucht, um etwa durch den Nachweis einer erheblichen Steigerung in demselben (normal als schwankend angegeben zwisohen $1 / 4$ und 1 pro mille) auf die entzindliche Natur der Fltissigkeit schließen zu durfen. Die Angaben der Autoren (Leube, Sahli, Oppenheim) uber den diagnostischen Wert dieses Symptoms für die Diagnose einer Meningitis serosa sind freilich noch sebr unsicher und schwankend, offenbar weil bis jetzt noch niemand ein eignes, etwas größeres Untersuchungsmaterial gehabt hat. -

Schon lange bekannt sind die bei Otitis media acuta der Kinder mitunter vorkommenden schweren meningitisehen Sym. ptome, die ich auch nach Eintritt der Pubertăt noch öfter gesehen habe und zuerst in meinem Lehrbuch der chirurgischen Krankheiten des Ohres vor 25 Jahren bescbrieben und auch dort mit einem typischen Beispiel belegt habe.

Ein 17 jähriger Jüngling mit akuter Otitis media zeigte trotz bereits erfolgter spontaner Perforation des einen Trommelfells und reichlichem Eiterabfluß aus dem Gehörgange sehwere, cerebrale Symptome bei hohem Fieber. Es bestanden tiefer Sopor, Myosis, Delirien ohne Unterlaß. Diese Symptome gingen aber nicht von dem vermeintlich allein erkrankten Ohre, in welchem das Trommelfell vom Eiter durehbrochen war, aus, sondern von dem $\mathrm{zweiten}$ Ohre, uber welches, so lange der Kranke noch bei Bewußtsein gewesen war, nie geklagt war. Das Trommelfell dieses zweiten Obres war durch reichliches eitriges Exsudat in der Paukenhöhle stark vorgebaucbt. Nachdem durch Paracentese dem Eiter Abfluh geschaffi war, verschwanden die meningitischen Symptome schnell und der Kranke genas vollständig. Ähnliche Fälle habe ich seitdem mehrfach erlebt, und von Dr. Hick s ${ }^{1}$ ) ist sogar ein Fall mitgeteilt worden, wo bereits Mydriasis, Ptosis und Parese des einen Beines bestanden hatten, also Symptome, die noch sicherer auf das Bestehen einer eitrigen Meningitis hinzudeuten sehienen, und dennoch durch die Paracentese des Trommelfells sehnelle und vollständige Heilung erfolgt sein soll.

Man hat versucht, sich diese meningitischen Symptome zu erklären durch toxische Einwirkung der von den Bakterien ausgeschiedenen Toxine, welche in den Hirnhäuten und der Hirnsubstanz eine der Räckbildung fähige Entzündung veranlassen

1) Amer. Journal of otology 1. p. 205. 
sollen. Die Bildungsstätte der giftigen Toxine sollen die Eiterungen im Schläfenbein sein. Das anffallend schnelle Schwinden dieser psendomeningitisehen Symptome nach der Paracentese macht diese Erklärung wenig wahrscheinlich.

Ebenso bekannt sind die häufig vorkommenden Komplikationen der ehronischen eitrigen Otitis mit tuberkulöser Meningitis. Hier kann es, wenn sich Tuberkelbazillen im Eiter nicht nachweisen lassen und anch der ja doch immerhin seltene Befund von Chorioidealtuberkeln in der Peripherie des Augenhintergrundes nicht die Diagnose aufklären kann, zweifelhaft bleiben, ob die zur Ohreiterung hinzutretenden meningitischen Symptome veranlabt sind dureh Meningitis purulenta oder Meningitis tubereulosa. In einzelnen Fällen kann dies wirklich recht sehwierig sein, wenigstens bei kurzer Beobachtungsdauer. Ganz unmöglich kann die Diagnose werden, wenn sich bei chroniseher Otorrhoe eines tuberkulös belasteten und mit Knochentuberkulose an den Extremitäten resp. Narben von solchen behafteten Individuums Symptome eines intrakraniellen Leidens hinzugesellen, die auf Hirnabszeb oder Hirntumor hindeuten. Einen bemerkenwerten Fall der Art habe ich bei einem 12jährigen Knaben aus Rußland 1893 beobachtet und bescbrieben ${ }^{1}$ ). Hier ergab der Sektionsbefund ein großes Gliosarcoma cerebelli neben hochgradigem Hydrocephalus internus chronicus.

Daß bei Meningitis purulenta fulminans ex otitide eine Apoplexia cerebri als Todesursache fälschlicherweise diagnostiziert wird, wie ich einige Male erlebt habe, kommt in der generellen Praxis gewiß häufiger vor, sobald jede Anamnese fehlt und das Ohr nicht untersueht wird. Die Symptome können in der Tat wegen des Coma und der halbseitigen Körperläbmung sehr ähnlioh sein. Durch Lumbalpunktion würde jetzt vielleicht die Diagnose zu sichern sein, wenn durch dieselbe völlig klarer Liquor entleert wird.

In meiner Klinik haben wir aber auch den umgekehrten Fall gesehen 2), wo bei einem kräftigen jungen Mädohen von etwa 18 Jahren einige Wochen nach operierter Otitis (Totalaufmeißelung) plötzlicher Tod ganz unerwartet in der Nacht eintrat, und die Sektion als Todesursache eine frische Apoplexie im Thalamus optious ergab ohne Spur von Meninigitis.

1) S. dieses Archiv, Bd. 38 S. 292.

2) Publiziert in diesem Archiv.

Archiv f. Ohrenheilkunde. Bd. 80. 
Ebenso iberrasehend war der Sektionsbefund, wo bei ausgesprochen meningitischen Symptomen und bei anscheinend zweifelloser Diagnose eitriger Meningitis ex otitide (tiefer Sopor klonische und tonische Konvulsionen) usw.) der Sektionsbefund (s. dieses Archiv, Bd. 78 S. 229) nichts ergab, als hochgradige Anaemia universalis, besonders auch des Gebirns. Seitdem wir die Lumbalpunktion als diagnostisehes Mittel haben, werden ähnliche Irrtimer leichter $\mathrm{zu}$ vermeiden sein. Jedoch hat uns die neueste Zeit gelehrt, daß ausnahmswe ise auch Fälle mit leicht getribtem Liquor (durch mäßige Lenkocytenvermehrung bei fehlendem Nachweis von Streptokokken), noch heilungsfahig sind, und daß derartige leichte Trübungen also noch nicht als sichere Anzeichen einer unbedingt lethalen purulenten Meningitis angesehen werden durfen. Auch bei Pneumonie und bei abgeschlossenen Hirnabszessen, die noch nicht durchbrochen sind nach der Hirnoberfläche oder in die Ventrikel und eventuell also noch operabel sind, sollen solche leichte Trubungen des Liquors vorkommen.

Stärkere Trübungen mit stark vermehrten polynukleären Leukocyten und Streptokokken, oder eitrige Trubungen lassen dagegen nach meiner bisherigen Erfahrung jede Operation als hoffnungslos erscheinen. 